Western University

Scholarship@Western

Physics and Astronomy Publications

Physics and Astronomy Department

$1-2014$

\title{
Surface Plasmon Resonance Sensing Properties of a 3D Nanostructure Consisting of Aligned Nanohole and Nanocone Arrays
}

Mohamadreza Najiminaini

Western University

Erden Ertorer

Western University

Bozena Kaminska

Simon Fraser University

Silvia Mittler

Western University, smittler@uwo.ca

Jeffrey J.L. Carson

Western University

Follow this and additional works at: https://ir.lib.uwo.ca/physicspub

Part of the Astrophysics and Astronomy Commons, and the Physics Commons

Citation of this paper:

Najiminaini, Mohamadreza; Ertorer, Erden; Kaminska, Bozena; Mittler, Silvia; and Carson, Jeffrey J.L., "Surface Plasmon Resonance Sensing Properties of a 3D Nanostructure Consisting of Aligned Nanohole and Nanocone Arrays" (2014). Physics and Astronomy Publications. 40.

https://ir.lib.uwo.ca/physicspub/40 
Analyst, 139 (2014) 1876-1882

\section{Surface plasmon resonance sensing properties of a 3D nanostructure consisting of aligned nanohole and nanocone arrays}

Mohamadreza Najiminaini ${ }^{1,2,3}$, Erden Ertorer ${ }^{4,5}$, Bozena Kaminska ${ }^{2}$, Silvia Mittler ${ }^{4}$ and Jeffrey J.L. Carson ${ }^{1,3, *}$

$$
\begin{aligned}
& \text { 1maging Program, Lawson Health Research Institute, St. Joseph's Health Care, London, ON, Canada } \\
& { }^{2} \text { The School of Engineering Science, Simon Fraser University, Burnaby, BC, Canada } \\
& { }^{3} \text { The Department of Medical Biophysics, Schulich School of Medicine and Dentistry, University of Western Ontario, London, ON, Canada } \\
& { }^{4} \text { The Department of Physics and Astronomy, University of Western Ontario, London, ON, Canada } \\
& { }^{5} \text { Biomedical Engineering Program, Faculty of Engineering, University of Western Ontario, London, ON, Canada } \\
& \text { *Correspondence: jcarson@lawsonimaging.ca }
\end{aligned}
$$

\section{Abstract}

Molecular surface plasmon resonance (SPR) sensing is one of the most common applications of an array of periodic nanoholes in a metal film. However, metallic nanohole arrays (NHAs) with low-hole count have lower resolution and SPR sensing performance compared to NHAs with high-hole count. In this paper, we present a compact three-dimensional (3D) plasmonic nanostructure with extraordinary optical transmission properties benefiting from surface plasmon matching and enhanced localized surface plasmon coupling. The 3D nanostructure consisted of a gold film containing a NHA with an underlying cavity and a gold nanocone array (NCA) at the bottom of the cavity. Each nanocone was aligned with the nanohole above and the truncated apex of each nanocone was in close proximity $(100 \mathrm{~nm})$ to the gold film. The NHA-NCA structures outperformed conventional NHA structures in terms of bulk sensitivity and Figure of Merit (FOM). Furthermore, the NHA-NCA structure with 525-nm periodicity was capable of sensing streptavidin down to $2 \mathrm{nM}$ exhibiting a 10-fold increase in streptavidin sensitivity compared to conventional NHA structures. The sensitivity and performance of the 3D nanostructure can be further improved by exploiting multiplexing methods in combination with stable light sources and detection systems. 


\section{Introduction}

Surface Plasmon Resonance (SPR) sensing has been recognized as one of the most important applications of metallic nanostructures and has been exploited for the detection of molecules [1,2]. The surface plasmon (SP), or physically exact surface plasmon polariton (SPP), is a collective oscillation of the free electron gas at the interface between a metal and a dielectric; it is a longitudinal wave propagating along the metal-dielectric interface producing evanescent fields in the metal and in the dielectric [3]. The SPR sensing principle is based on a change in the coupling conditions between an incident light beam and the SP due to a change in the surrounding dielectric material, e.g. resulting from material adhesion onto the metal, changing the refractive index architecture [4]. Localized surface plasmon resonance (LSPR), on the other hand, is the collective oscillation of the free electron gas in a metal nanoparticle leading to an absorption band whose location also depends on the refractive index architecture around the nanoparticle [5].

A periodic array of nanoholes in a metal film allows wave vector matching between an incident light on a nanohole array (NHA) and the SP waves existing at the interface between a metal and a dielectric. This effect results in an interaction of the light with the SP waves [6]. The excitations of SP waves by an incident light on NHAs generate transmission based SPRs or so called extraordinary optical transmissions (EOTs). The EOTs of a NHA depend greatly on the dielectric functions of the metal and the dielectric materials and geometrical parameters of the NHA (hole spacing, hole arrangement and hole size) [7]. However, an individual nanohole can produce transmission based LSPR, which is associated to an interaction of the incident light with an LSP around the nanohole. Both an individual nanohole and a NHA have enhanced electric field intensity around each nanohole at the corresponding resonance wavelengths. 
Both LSPR of a single nanohole and SPRs of a NHA were extensively exploited in LSPR or SPR-based detection of chemicals and molecules $[2,6]$. However, in the context of the NHA literature, SPR sensing performance of a NHA were evaluated by bulk sensitivity (nm per refractive index unit (RIU)) and figure of merit (FOM (1/RIU)). The bulk sensitivity is well known as SPR peak shift (nm) of a NHA per RIU and the FOM is recognized as the bulk sensitivity over the peak width (FWHM) of the SPR. Nevertheless, the FOM terminology for nanoparticles and a signal nanohole is defined differently and it is calculated based on LSPR peak shifts per RIU. In this context, we use performance metrics associated to the NHA and name all resonances SPR despite some of them being local.

Kretschmann-based SPR sensing remains the most sensitive method and has motivated several groups to study methods to improve the bulk sensitivity and FOM of NHAs [8-14]. Based on recent studies, SPR performance of NHAs was improved by narrowing the resonance peak width, incorporating a smooth metal surface, index matching the substrate and the sensing material, and using design strategies to increase the electric field near the nanoholes. For example, it was reported for a bulk SPR sensing experiment (where the environmental refractive index is changed) that the bulk sensitivity of a NHA in a gold film reached Kretschmann-like performance when the substrate was index-matched to the analyte, the gold NHA was fabricated with an ultra-smooth surface, and elliptical hole shapes were used [9]. Further improvements to the bulk sensitivity and FOM of metallic NHAs were achieved by fabricating the NHA with dimensions larger than the excited SP propagation length, which constructively enhanced the excited SP interactions thereby improving the transmission efficiency of the resonance and narrowing the resonance peak width [15]. Various transmission resonances of a NHA are associated to different SP excitation modes based on the relationship between the scattering 
order of the holes, similar to Bragg diffraction in a crystal [6]. The transmission resonances are therefore classified with a number combination $(x, y)$ regarding the plane of the NHA. The bulk sensitivity of various transmission resonance modes of a NHA are different and the maximum bulk sensitivity has been achieved for the $(1,0)$ transmission resonance of a NHA [13]. For example, the $(1,0)$ resonances of large NHAs were reported to have bulk sensitivity as high as $600 \mathrm{~nm}$ per refractive index unit (nm/RIU) $[8,16,17]$, while the bulk sensitivity and FOM of a NHA with low hole count (i.e. less than 100) for the $(1,0)$ resonance was $286 \mathrm{~nm} / \mathrm{RIU}$ and 4.1 (1/RIU), respectively [16].

Some attempts to improve bulk sensitivity of a low-hole-count NHA were tested by incorporating Bragg reflectors into the structure [12,18]. Our group recently observed that a lowhole-count NHA with a large integrated cavity provided a 1.6-fold improvement in bulk sensitivity compared to a NHA structure without a cavity, due to dynamic SP matching between the top and the bottom of the NHA [14]. The matching between SP propagating along the top and the bottom of the NHA were very similar due to the symmetric gold structure in the case with the cavity and therefore enhanced the transmission resonance by multiple fold [19]. Threedimensional (3D) metallic nanostructures consisting of co-registered nanoholes and nanodisks have recently been reported and shown to exhibit transmission resonances due to coupled LSP interaction between nanoholes and nanodisks through enhanced electric fields [20]. In bulk SPR sensing, the 3D metallic nanostructures had a higher bulk sensitivity than low-hole-count NHAs with cavities, but the resonance peak width was broader [20]. Building upon these prior results, the objective here was to design a new 3D nanostructure with improved bulk sensitivity and FOM, where transmission resonances resulted from the coupling and interaction of both LSP and SPP. The approach was to devise a structure with enhanced electric field near each nanohole by 
incorporating dynamic-SP matching and one flat metal surface. We fabricated a 3D metallic nanostructure consisting of a NHA in a gold film with an underlying cavity carrying a gold nanocone array (NCA) at its bottom (Figure 1). The gold NCA was responsible for generating strong LSP interactions between each nanohole and nanocone as well as providing for coupling between the SPP and the LSP of the NHA and NCA, respectively.

We performed both simulations and experimental studies to determine the transmission resonance properties of the NHA-NCA structures. Various NHA-NCA structures with different geometries were fabricated and tested as bulk SPR sensors. The NHA-NCA structures were functionalized with a binary mixture of $\mathrm{OH}$-terminated and biotinylated thiols and evaluated as molecular SPR sensors for streptavidin.

We employed an electron beam lithography and liftoff fabrication process to create the NHA in an 80-nm-thick gold film on a Pyrex substrate. Extensive description of the NHA fabrication methodology has been reported previously [21]. Prior to 80 -nm gold deposition and the liftoff process, a 6-nm-thick Ti adhesion layer was evaporated on to the substrate using electron beam deposition. After fabrication, the NHA sample was immersed in Ti etchant (TFTN, Transene company, Inc.) for 70 seconds to remove the Ti adhesion layer and create a 250 -nm-deep cavity within the Pyrex substrate beneath the NHA in the metal film. A 150-nm-thick gold film was deposited by electron beam evaporation on top of the freestanding gold NHA and resulted in a thickening of the gold film to $230 \mathrm{~nm}$ and formation of a NCA at the bottom of the cavity. A schematic of the fabrication processes before and after gold deposition is shown in Figure 1 (a). We fabricated various 3D nanostructures with different hole periodicities and diameters. All devices had a 250-nm-deep cavity and 150-nm-tall nanocones. The nanoholes and nanocones had periodicities between $375 \mathrm{~nm}$ and $550 \mathrm{~nm}$ in increments of $25 \mathrm{~nm}$. The ratio between overall 
nanohole area to filled gold area (i.e. square of hole periodicity) was 0.096 for all 3D nanostructures. The lowest and highest hole diameters for the 3D nanostructures were $131 \mathrm{~nm}$ and $192 \mathrm{~nm}$, respectively. The periodicities were selected such that the main transmission resonances were located between $400 \mathrm{~nm}$ and $850 \mathrm{~nm}$ to match the spectral range of the characterization setup. The area of each array was about $25 \mu \mathrm{m}^{2}$ and each array was repeated in a 2D grid of 7 by 7 with $10-\mu \mathrm{m}$ spacing between adjacent arrays to minimize surface plasmon crosstalk [22]. Figure 1 (b) displays an SEM image of a NHA-NCA structure with 500-nm periodicity, a 230-nm-thick gold film, 150-nm-tall gold nanocones, and a 250-nm-deep cavity. The SEM images revealed that each nanocone was right circular in geometry and had a truncated apex. The base diameter of each nanocone was similar to the nanohole diameter measured prior to deposition of the 150-nm-thick layer of gold. The diameter of the truncated apex of each nanocone was approximately half of the diameter of the base. The diameter of the truncated nanocone apex was dependent on the gold deposition rate (data not shown). The diameter of each nanohole at the top and the bottom surfaces of the gold film were similar in diameter to the base of each gold nanocone. In addition, the nanohole diameter decreased from both the top and the bottom surfaces to the middle of the gold film. 


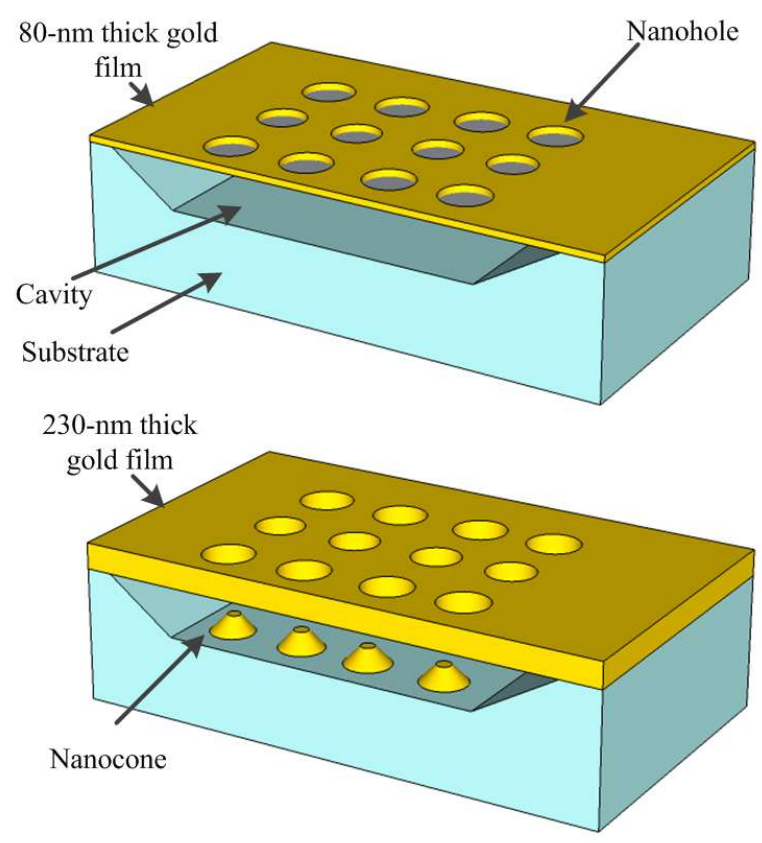

(a)

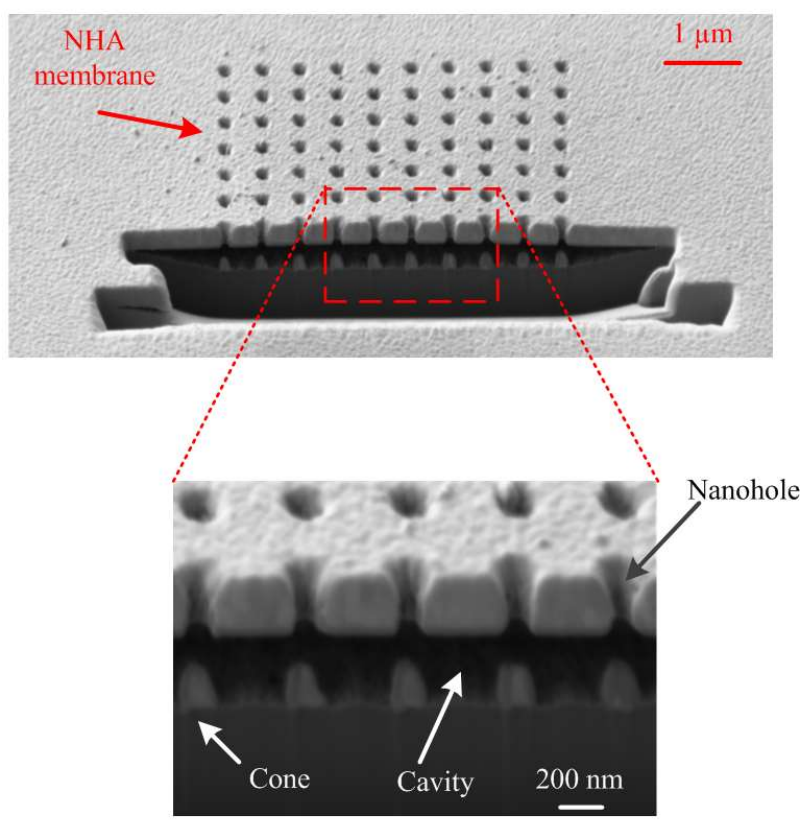

(b)

Figure 1. a) Schematic of a NHA in a gold film with a large cavity etched into the Pyrex substrate (top panel). A schematic of a NHA in a gold film with an underlying gold NCA, where each nanocone was axially aligned with the hole above and was formed by deposition of gold through the NHA from the top surface (bottom panel). b) SEM image of a NHA in a 230 -nm-thick gold film and a $150-\mathrm{nm}$-tall gold NCA with 500 -nm periodicity. The cavity depth was $250 \mathrm{~nm}$. The largest and smallest diameters of the holes and cones were $175 \mathrm{~nm}$ and $90 \mathrm{~nm}$, respectively.

We employed Finite Difference Time Domain (FDTD) simulations (Lumerical Inc., Vancouver, Canada) to compute the optical transmission properties of the NHA-NCA structures. In the simulation model, we used an incident plain wave propagating in the direction normal to the sandwich structure. Periodic and perfectly matched layer boundary conditions were used in $x y$ directions and at the $z$ boundaries, respectively. The minimum mesh size was three nanometers. Figure 2 (a) displays optical transmission spectra of NHA-NCA structures with 500-nm periodicity for a range of nanohole and nanocone diameters. The gold NCA had two main LSP absorption resonances, at $678 \mathrm{~nm}$ and $847 \mathrm{~nm}$, related to the truncated apex and the base, respectively as seen from the electric field distribution for each absorption peak (Figure 2 (b)). The highest electric field intensity (hot spot) was found at the perimeter of the truncated apex of the nanocone, which had a dipolar mode due to the excitation of LSP. The dipolar mode stems 
from strong electric field distributions (hot spots) on the two opposite sides of the nanocone perimeter (data not shown). Based on previous work on similar structures [23,24], it has been shown that a strong electric field is confined to the tip of the nanocone. As a result, the absorption peak at $678 \mathrm{~nm}$ from the truncated surface of the gold nanocone was due to the vertical LSP excitations of the nanocone, which eventually resulted in the LSP absorption at the apex. Moreover, the simulation intensity maps for wavelengths between $550 \mathrm{~nm}$ and $780 \mathrm{~nm}$ revealed the presence of a confined electric field at the apex of the nanocone. Nevertheless, amplitudes of energy confinement at the apex of the nanocone were not the same for different wavelengths. The variations in the localized electric field intensity at the apex of the nanocone were likely due to size-dependence of vertical LSP excitations of the nanocone as well as the wavelength-dependent absorption properties of gold.

The simulated optical transmission of the NHA-NCA structure demonstrated the existence of two transmission resonance peaks that were related to the $(-1,0)$ and $(1,1)$ SP excitation modes. The electric field maps at both resonances showed pronounced LSP coupling between the apex of the nanocone and the bottom aperture of the nanohole as shown in Figure 2 (b) (right panel). The LSP coupling between the nanocone and nanohole was stronger for the $(-1,0)$ resonance than the $(1,1)$ resonance, which was due to the deeper SP decay length from the metal film into the dielectric at longer wavelengths [3]. In addition, LSP absorption was apparent above $800 \mathrm{~nm}$ due to the base of the nanocone. Furthermore, the NHA-NCA was responsible for SP-light coupling and an evanescent transfer of SP-light energy to the other side of the metal film. As a result, each of the $(-1,0)$ and the $(1,1)$ resonances had LSP and SPP characteristics. Also, due to inherent SPP properties of the resonances of the 3D nanostructure, both the $(-1,0)$ and the $(1,1)$ spectral resonance positions depended greatly on the spacing between the nanoholes. However, 
we observed that the $(-1,0)$ resonance was further divided into two resonances when the periodicity was larger than $500 \mathrm{~nm}$. The reason behind this phenomenon was the LSP interaction between nanohole and nanocone, which became dominant and generated two individual resonance peaks.

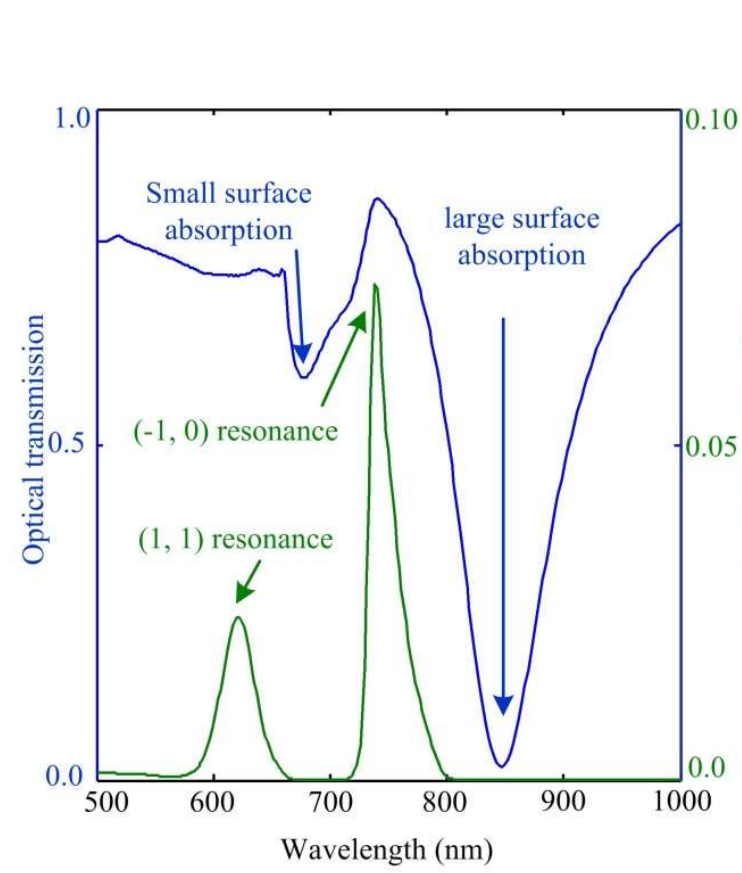

(a)
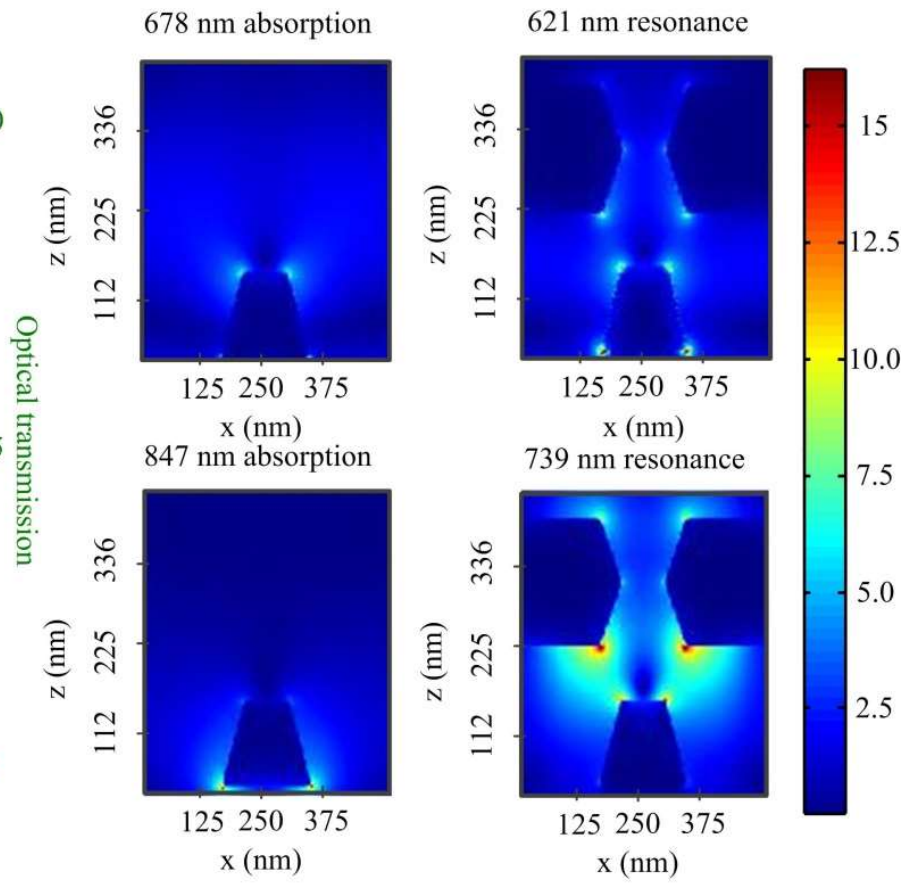

(b)

Figure 2. a) Simulated optical transmission spectra of a 150-nm-thick gold NCA with periodicity of $500 \mathrm{~nm}$. Each nanocone had an apex diameter of $87.5 \mathrm{~nm}$ and a base diameter of $175 \mathrm{~nm}$. The substrate had a refractive index of $\mathrm{n}$ $=1.474($ Pyrex, at $\lambda=587 \mathrm{~nm}$ ) and the surrounding material had a refractive index of $\mathrm{n}=1.330$ (water, at $\lambda=587$ $\mathrm{nm}$ ) (blue curve). Simulated optical transmission spectrum of a 230-nm-thick gold NHA membrane and a 150-nmthick gold NCA at the bottom of the 250-nm-deep cavity (green curve). The nanohole had a diameter of $90 \mathrm{~nm}$ (midpoint in film) and a diameter of $175 \mathrm{~nm}$ (top and bottom of film). b) Electric field distribution of a nanocone at absorption wavelengths of $678 \mathrm{~nm}$ (top left) and $847 \mathrm{~nm}$ (bottom left). Electric field distribution of a combined nanohole and nanocone at transmission resonances located at $621 \mathrm{~nm}$ (top right panel) and $739 \mathrm{~nm}$ (bottom right panel).

To measure the optical transmission of the $3 \mathrm{D}$ gold nanostructures, we employed an inverted microscope (TE300, Nikon, Japan) equipped with a photometer, monochromator, and photomultiplier-based detector (PTI, Birmingham, NJ). The incident illumination (Halogen lamp, $12 \mathrm{~V}, 100 \mathrm{~W}$, Ushio Inc., Tokyo, Japan) was focused on to each 3D nanostructure array using the bright field condenser. The illumination angle was controlled to within $\pm 6^{\circ}$ of normal 
incidence. The transmitted light was collected with a 20x objective (NA $=0.45$; model\# 93150 , Nikon). A region of interest was selected with the aperture controls on the photometer. For each spectral scan, the region of interest was chosen to allow the transmitted light from one set of 25 identical 3D nanostructure arrays through to the monochromator and detector. This methodological step resulted in average transmission spectra representative of many arrays and served to enhance the signal-to-noise ratio. Transmission spectra of 3D arrays with 475-nm periodicity and 250-nm-cavity depth were measured when each liquid refractive index standard between 1.30 and 1.39 in increments of 0.03 was applied to the top surface (Figure 3 (a)). Because of capillary forces, the liquid filled the cavity. Similar to simulation results, the $(-1,0)$ and the $(1,1)$ transmission resonances were observed in the measured spectra. However, an additional weak transmission resonance was observed between the $(1,1)$ and $(-1,0)$ resonances and was likely related to the $(0, \pm 1) \mathrm{SP}$ excitation mode. The weak resonance was apparent in the measured spectra due to the wide range of incident illumination angles $\left( \pm 6^{\circ}\right)$ compared to normally incident illumination used in the simulations. In a similar manner to the simulation results, the 3D nanostructure arrays with $525-\mathrm{nm}$ and $550-\mathrm{nm}$ periodicities revealed a double resonance near the expected position of the $(-1,0)$ resonance. The double resonance may have been related to a stronger LSP interaction between nanocone and nanohole at longer wavelengths, where the second resonance at longer wavelength was associated with the LSP interaction between each nanohole and nanocone.

Using the transmission spectra from each set of 3D nanostructure arrays, estimates of resonance peak width and bulk sensitivity were determined. The bulk sensitivity was estimated from the slope of the line of best fit of the resonance position versus refractive index. For the 3D nanostructures with $475-\mathrm{nm}$ periodicity, the measured $(-1,0)$ and $(1,1)$ resonance peak widths 
(FWHM) were $40 \mathrm{~nm}$ and $56 \mathrm{~nm}$, respectively. Also, the bulk sensitivities for the $(-1,0)$, the $(1$, 1) and the $(0, \pm 1)$ resonances were $440 \mathrm{~nm} / \mathrm{RIU}, 280 \mathrm{~nm} / \mathrm{RIU}$ and $460 \mathrm{~nm} / \mathrm{RIU}$, respectively. The FOM for the $(-1,0)$ and the $(1,1)$ resonances were 11 1/RIU and $51 /$ RIU, respectively. The bulk sensitivities related to the $(-1,0)$ resonance of the 3D nanostructures are shown in Figure 3 (a) as a function of periodicity. As expected from earlier experimental and theoretical analysis (see Refs $[13,14])$, the bulk sensitivity of the 3D nanostructures increased with increasing periodicity. The maximum bulk sensitivity (593 nm/RIU) and FOM (12.90 1/RIU) was found for the 3D nanostructures with 550-nm periodicity. Therefore, for bulk SPR sensing, the 3D nanostructure had a larger than 2-fold higher bulk sensitivity and in excess of 3-fold greater FOM compared to reported results (see Refs. $[12,16]$ ) for conventional NHAs with low hole count.

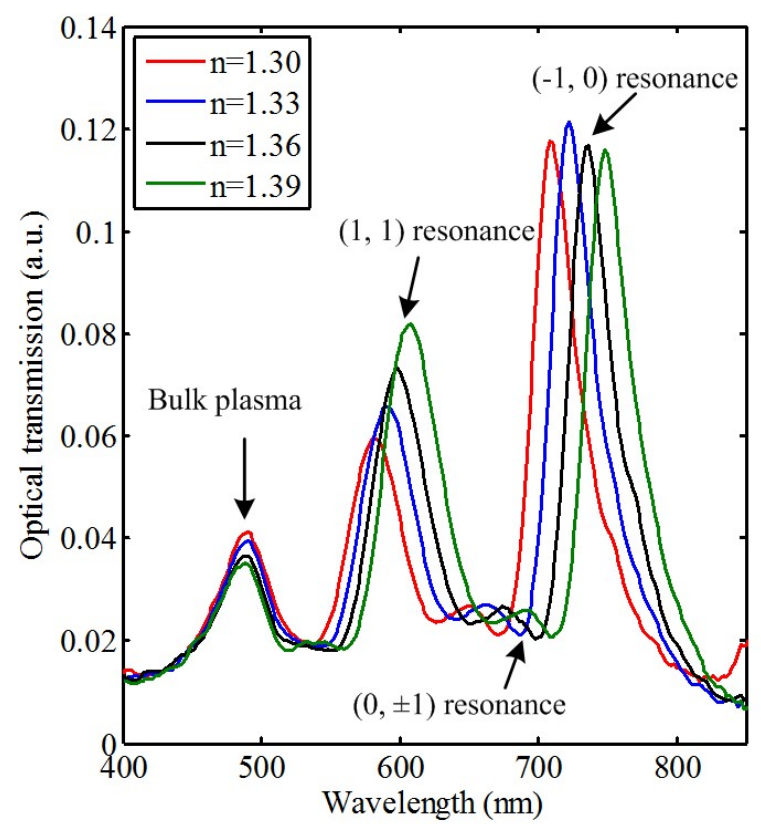

(a)

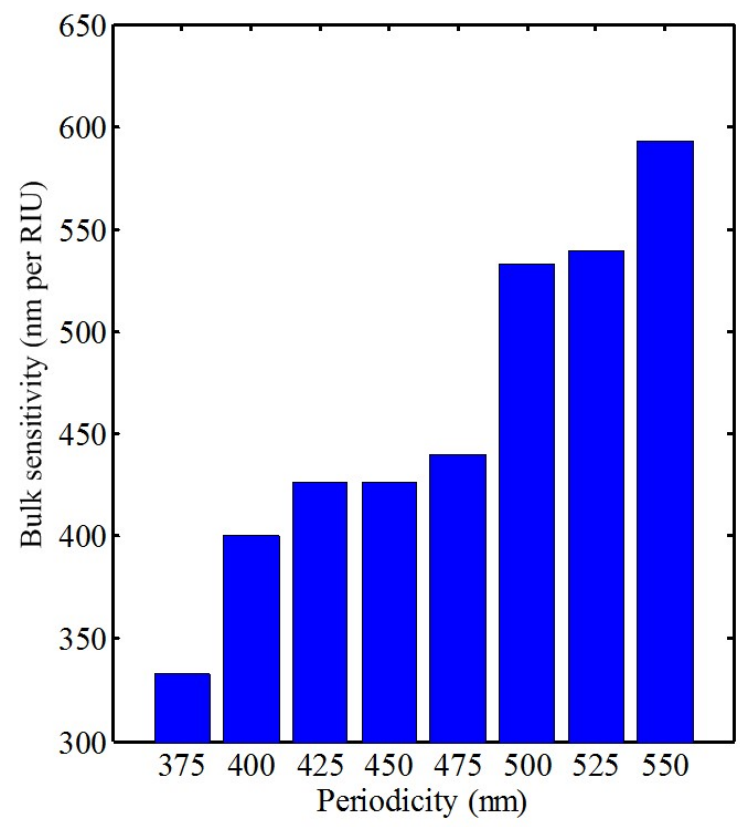

(b)

Figure 3. a) Optical transmission spectra of a NHA-NCA structure taken when one of four refractive index standards $(1.30,1.33,1.36$, and 1.39 at $\lambda=589 \mathrm{~nm})$ was placed on top of the surface. The NHA and NCA periodicity was 475 $\mathrm{nm}$. The cavity beneath the NHA was $250-\mathrm{nm}$ deep. The apex and base of each nanocone had diameters of $84 \mathrm{~nm}$ and $170 \mathrm{~nm}$, respectively. The gold film was 230-nm thick. The height of each nanocone was $150 \mathrm{~nm}$. b) Measured bulk sensitivity of the NHA-NCA structure as a function of NHA and NCA periodicity. Bulk sensitivity was estimated by the slope of the wavelength of the $(-1,0)$ resonance peak versus the refractive index of the medium above the top surface as shown in panel (a). 
Functionalized 3D nanostructures were evaluated in SPR sensing of streptavidin in PBS buffer. The gold surfaces of the 3D nanostructures were functionalized with a binary mixture of an $\mathrm{OH}-$ terminated and biotinylated thiol to offer biotin as recognition sites for streptavidin in the right amount and to allow streptavidin binding without steric hindrance (see schematic diagram in Figure 4 (a)). Functionalization was achieved by immersing each device into an anhydrous ethanol solution composed of $0.45-\mathrm{mM}$ 11-mercaptoundecanol (Sigma-Aldrich, Ontario, Canada) and 0.05-mM biotinylated thiol (NanoScience Chemicals, Phoenix, AZ, USA) for 24 hours to create a self-assembled monolayer (SAM) with biotin moieties. The device was then rinsed with ethanol and dried with nitrogen. Finally, the sample was placed in PBS buffer, streptavidin (Rockland Inc., PA, USA) was added. Measurements were performed within 5 minutes.

The $(-1,0)$ resonance peak shifts of each 3D nanostructure array in response to $2 \mathrm{nM}, 20 \mathrm{nM}$, 200 nM, 2000 nM, and 3500 nM streptavidin were measured and are shown in Figure 4 (b). In order to determine a precise $(-1,0)$ resonance position from each spectrum, we employed Gaussian curve fitting on the $(-1,0)$ resonance peak. All measurements were repeated in triplicate and the results are reported in Figure 4 (b) as mean \pm 1 standard deviation (SD). Furthermore, the detection confidence was calculated for each 3D nanostructure based on the mean value from triplicate PBS measurements (blue solid line in Figure 4 (b)) \pm 3 times the average noise (blue dashed lines in Figure 4 (b)). The limit of detection (LOD) of a 3D nanostructure was the lowest detectable concentration of streptavidin where there was enough certainty (i.e. above detection confidence). Considering all devices, the $(-1,0)$ resonance peak red-shifted as the concentration of streptavidin increased. The largest spectral resonance shift was observed when the concentration was increased from $200 \mathrm{nM}$ to $2000 \mathrm{nM}$. However, the 
size of the resonance shift in response to streptavidin was not consistent across all devices. For example, the $(-1,0)$ resonance shift between PBS base line and 3500-nM streptavidin was largest for the 3D nanostructure with $375-\mathrm{nm}$ periodicity. This effect may have been due to the smaller nanohole and nanocone dimensions for the 375 -nm periodicity structure, which resulted in more significant changes in LSP coupling between nanocones and nanoholes due to the smaller size of nanohole and nanocone. However, 3D nanostructures with larger periodicities (i.e. $500 \mathrm{~nm}$ and $525 \mathrm{~nm})$ had larger $(-1,0)$ resonance shifts for the lowest concentrations of streptavidin. Moreover, the lowest concentration of streptavidin $(2 \mathrm{nM})$ was only detectable with the 3D nanostructure with 525-nm periodicity, where the detection certainty of the LOD was calculated at $95 \%$ confidence interval [25]. This finding substantiates the observation of higher bulk-SPR sensing for devices with larger periodicities compared to devices with smaller periodicities (e.g. see Refs [13]).

Previously, large conventional NHAs with $650-\mathrm{nm}$ periodicity and $400-\mu \mathrm{m}^{2}$ area were used to detect streptavidin binding by monitoring the spectral SPR shift [17]. The average resonance peak shift for high concentrations of streptavidin $(20 \mu \mathrm{M})$ was found to be between $3.5 \mathrm{~nm}$ and 4 $\mathrm{nm}$. In another study, a multiplexed arrangement of NHAs, each with an area of approximately $40 \mu \mathrm{m}^{2}$ and providing a variety of resonance positions, were shown to improve the limit of streptavidin detection down to $25 \mathrm{nM}$ with the use of a stable laser source and cooled CCD camera [10]. In comparison with these previous studies, our 3D nanostructure was capable of sensing a 10 -fold lower streptavidin concentration $(2 \mathrm{nM})$ without the use of a laser or a cooled CCD camera. Therefore, it is expected that the detection limit of streptavidin with our 3D nanostructure could be reduced below $2 \mathrm{nM}$ by implementing a stable light source and a cooled detector. 


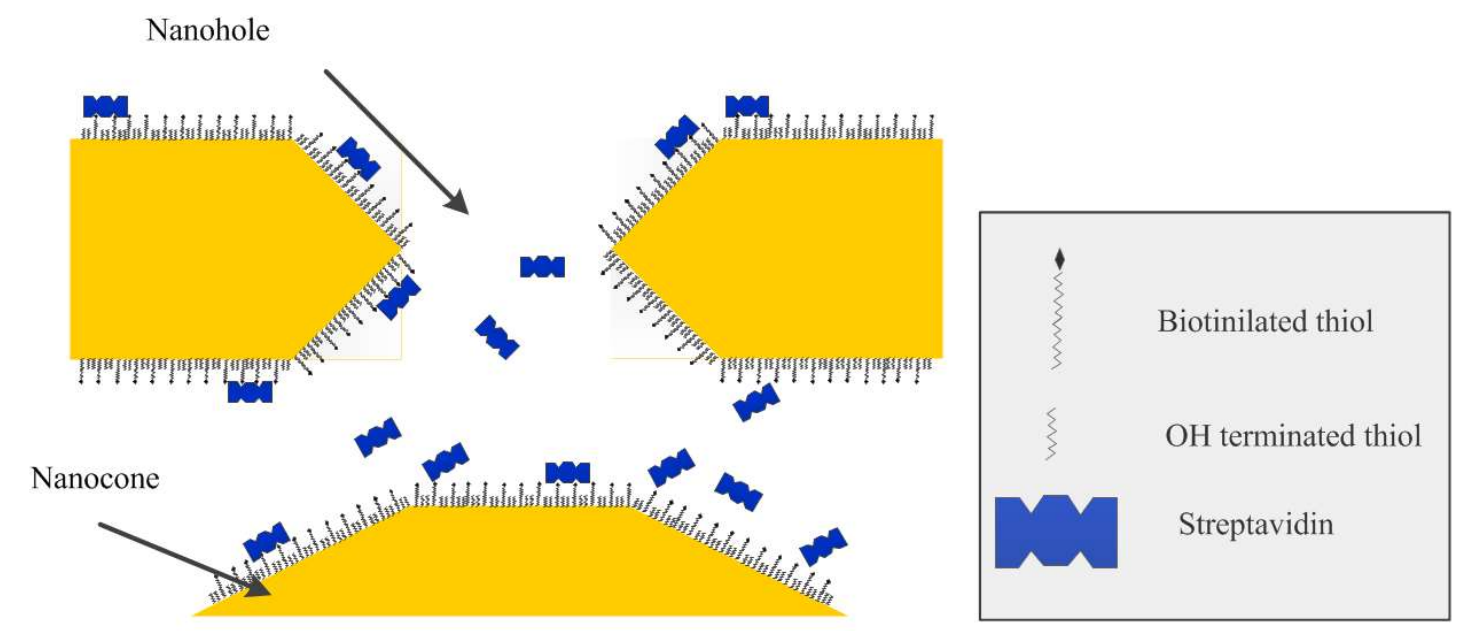

(a)
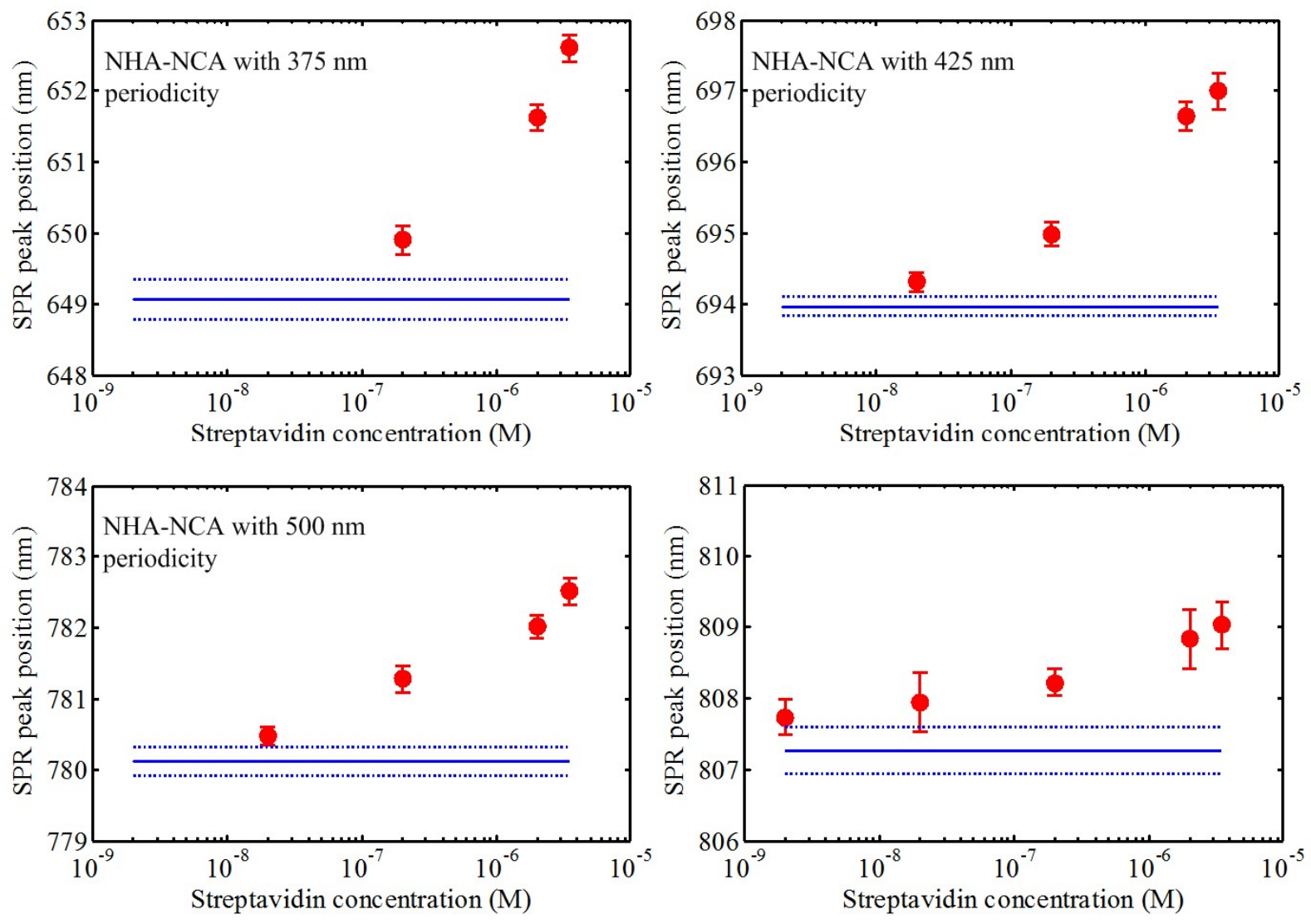

(b)

Figure 4. a) Schematic of a nanohole and adjacent nanocone showing the functionalized surfaces. Streptavidin molecules bind to biotinylated thiol. b) The SPR response of functionalized NHA-NCA structures to streptavidin. Each panel represents the SPR peak position for 25 identical nanostructure arrays with a periodicity indicated in the top left corner versus the streptavidin concentration in solution. The red error bars represent \pm 1 standard deviation 
computed from three different measurements for each data point. The solid blue line represents the base line from PBS measurements (mean value among triplicate PBS measurements) and the dashed blue lines represents \pm 3 times the standard deviation of the PBS measurements representative of the detection threshold.

Based on the bulk sensitivity measures, the NHA-NCA structures offer improved performance compared to conventional NHAs. Improved performance is likely due to a combination of factors. First, the NHA-NCA structure benefits from dynamic surface plasmon matching between the top and bottom surfaces of the gold NHA membrane due to the presence of the cavity [14]. Second, the bottom surface of the NHA membrane has an ultra-smooth metal surface, which resulted from the polished surface of the Pyrex substrate (with roughness parameter, $\mathrm{Ra}<15 \AA$ ) prior to etching. However, the top surface roughness of the NHA was about $1.9 \mathrm{~nm}$ as reported previously in Ref [21]. Third, the highly localized electric field between the nanohole and the nanocone due to the LSP interactions enable high sensitivity of this region of the device to changes in refractive index of the intermediate dielectric materials. Fourth, there were no Ti adhesion layers in the NHA-NCA structure to reduce optical performance, which resulted in higher bulk sensitivity of the NHA-NCA structure as reported for NHAs in previous studies [21,26]. One disadvantage of the NHA-NCA structure is the lower transmission efficiency at the resonances compared to the resonance transmission efficiency of a conventional NHA. According to experimental results, the resonance transmission efficiency for the NHANCA was almost 42 -fold lower than the NHA structure prior to forming a NCA. Lower transmission was a consequence of the optical absorption properties of the nanocone, a thicker NHA gold film, smaller nanohole size, and nanocone shadowing effects on each incident nanohole [20].

In conclusion, a 3D nanostructure consisting of a metallic film with a NHA, a cavity beneath the NHA, and a NCA at the bottom of the cavity was designed, fabricated, and tested through simulations and experiments. Experimental measurements of the NHA-NCA structure revealed 
transmission resonances due to LSP interactions between the nanocones and nanoholes and SPP interactions related to the NHA. A series of NHA-NCA structures were studied in bulk SPR sensing and found to have higher bulk sensitivity and FOM compared to conventional NHAs. Furthermore, NHA-NCA structures were employed for the detection of streptavidin. Compared to conventional NHA structures, one of the NHA-NCA structures was able to reliably detect streptavidin down to $2 \mathrm{nM}$, which exceeded the sensitivity of multiplexed detection methods (25 $\mathrm{nM}$ ) [10]. In future work, a multiplexed SPR sensor based on an array of NHA-NCA structures can be designed to improve the LOD for biomolecules over the integration method used to evaluate NHA-NCA structures described in this work.

\section{Acknowledgements}

The authors thank Dr. Todd Simpson for his technical support at the Western Nanofabrication Facility at the University of Western Ontario (UWO). We also would like to thank Dr. Hao Jiang for helpful discussion about the biosensing results. This project was funded by grants from the Natural Sciences and Engineering Research Council of Canada (NSERC) to Dr. Bozena Kaminska and Dr. Jeffrey J. L. Carson. Dr. Mohamadreza Najiminaini was supported by the MITACS program. Dr. Silvia Mittler would like to thank the NSERC BiopSys Strategic Network for financial contributions.

\section{References}

1. J. N. Anker, W. P. Hall, O. Lyandres, N. C. Shah, J. Zhao, and R. P. Van Duyne, "Biosensing with plasmonic nanosensors," Nat Mater 7, 442-453 (2008).

2. T. Rindzevicius, Y. Alaverdyan, B. Sepulveda, T. Pakizeh, M. Kall, R. Hillenbrand, J. Aizpurua, and F. J. Garc Ãa de Abajo, "Nanohole Plasmons in Optically Thin Gold Films," J. Phys. Chem. C 111, 1207-1212 (2007).

3. H. Raether, Surface plasmons (Springer-Verlag, 1988). 
4. C. Valsecchi and A. G. Brolo, "Periodic Metallic Nanostructures as Plasmonic Chemical Sensors," Langmuir 29, 5638-5649 (2013).

5. T. Jensen, L. Kelly, A. Lazarides, and G. Schatz, "Electrodynamics of Noble Metal

Nanoparticles and Nanoparticle Clusters," Journal of Cluster Science 10, 295-317 (1999).

6. T. W. Ebbesen, H. J. Lezec, H. F. Ghaemi, T. Thio, and P. A. Wolff, "Extraordinary optical transmission through sub-wavelength hole arrays," Nature 391, 667-669 (1998).

7. F Przybilla and A Degiron and J-Y Laluet and C Genet and,T.W.Ebbesen, "Optical transmission in perforated noble and transition metal films," J. Opt. A-Pure Appl. Op. 8, 458 (2006).

8. A. A. Yanik, M. Huang, A. Artar, T. Chang, and H. Altug, "Integrated nanoplasmonicnanofluidic biosensors with targeted delivery of analytes," Appl. Phys. Lett. 96, 021101-3 (2010).

9. G. A. Cervantes Tellez, S. Hassan, R. N. Tait, P. Berini, and R. Gordon, "Atomically flat symmetric elliptical nanohole arrays in a gold film for ultrasensitive refractive index sensing," Lab Chip 13, 2541-2546 (2013).

10. H. Im, A. Lesuffleur, N. C. Lindquist, and S. Oh, "Plasmonic Nanoholes in a Multichannel Microarray Format for Parallel Kinetic Assays and Differential Sensing," Anal. Chem. 81, 28542859 (2009).

11. A. Lesuffleur, H. Im, N. C. Lindquist, K. S. Lim, and S. Oh, "Laser-illuminated nanohole arrays for multiplex plasmonic microarray sensing," Opt. Express 16, 219-224 (2008).

12. N. C. Lindquist, A. Lesuffleur, H. Im, and S. Oh, "Sub-micron resolution surface plasmon resonance imaging enabled by nanohole arrays with surrounding Bragg mirrors for enhanced sensitivity and isolation," Lab Chip 9, 382-387 (2009).

13. L. Pang, G. M. Hwang, B. Slutsky, and Y. Fainman, "Spectral sensitivity of two-dimensional nanohole array surface plasmon polariton resonance sensor," Appl. Phys. Lett. 91, 123112-3 (2007).

14. M. Najiminaini, F. Vasefi, B. Kaminska, and J. J. L. Carson, "Effect of surface plasmon energy matching on the sensing capability of metallic nano-hole arrays," Appl. Phys. Lett. 100, 063110-4 (2012).

15. F. Przybilla, A. Degiron, C. Genet, T. Ebbesen, F. de Léon-Pérez, J. Bravo-Abad, F. J. García-Vidal, and L. Martín-Moreno, "Efficiency and finite size effects in enhanced transmission through subwavelength apertures," Opt. Express 16, 9571-9579 (2008).

16. J. Henzie, M. H. Lee, and T. W. Odom, "Multiscale patterning of plasmonic metamaterials," Nat Nano 2, 549-554 (2007). 
17. A. De Leebeeck, L. K. S. Kumar, V. de Lange, D. Sinton, R. Gordon, and A. G. Brolo, "OnChip Surface-Based Detection with Nanohole Arrays," Anal. Chem. 79, 4094-4100 (2007).

18. R. Gordon and P. Marthandam, "Plasmonic Bragg reflectors for enhanced extraordinary optical transmission through nano-hole arrays in a gold film," Opt. Express 15, 12995-13002 (2007).

19. A. Krishnan, T. Thio, T. J. Kim, H. J. Lezec, T. W. Ebbesen, P. A. Wolff, J. Pendry, L. Martin-Moreno, and F. J. Garcia-Vidal, "Evanescently coupled resonance in surface plasmon enhanced transmission," Opt. Commun. 200, 1-7 (2001).

20. M. Najiminaini, F. Vasefi, B. Kaminska, and J. J. L. Carson, "A three-dimensional plasmonic nanostructure with extraordinary optical transmission," Plasmonics 8, 217-224 (2012).

21. M. Najiminaini, F. Vasefi, B. Kaminska, and J. J. L. Carson, "Optical resonance transmission properties of nano-hole arrays in a gold film: effect of adhesion layer," Opt. Express 19, (2011).

22. F. Vasefi, M. Najiminaini, B. Kaminska, and J. J. L. Carson, "Effect of surface plasmon cross-talk on optical properties of closely packed nano-hole arrays," Opt.Express 19, 2577325779 (2011).

23. A. V. Goncharenko, M. M. Dvoynenko, H. Chang, and J. Wang, "Electric field enhancement by a nanometer-scaled conical metal tip in the context of scattering-type near-field optical microscopy," Appl. Phys. Lett. 88, 104101 (2006).

24. A. Mohammadi, F. Kaminski, V. Sandoghdar, and M. Agio, "Fluorescence Enhancement with the Optical (Bi-) Conical Antenna," J. Phys. Chem. C 114, 7372-7377 (2010).

25. D. Armbruster, M. Tillman, and L. Hubbs, "Limit of Detection (LOD)/Limit of Quantitation (LOQ): Comparison of the Empirical and the Statistical Methods Exemplified with GC-MS Assays of Abused Drugs," Clin. Chem. 40, 1233-1238- (1994).

26. M. Najiminaini, F. Vasefi, B. Kaminska, and J. J. L. Carson, "Nano-hole array structure with improved surface plasmon energy matching characteristics," Appl. Phys. Lett. 100, 043105 (2012). 